Achtung

Bitte informieren Sie sich frühzeitig, ob der für Sie interessante Termin stattfindet und kontaktieren Sie bei Fragen direkt den Veranstalter.

\section{FEBRUAR 2021}

Mannheim, 26.-27. Februar 2021 Geburtshilfe im Dialog Reschedule HYBRID

Fortbildungskongress, Forum für den Dialog in der Chinesischen Medizin www.tcm-im-dialog.de

Berlin, 28. Februar-02. März 2021

VegMed - Medizinischer Fachkongress zu pflanzenbasierter Ernährung ONLINE

ProVeg e.V., Physicians Association for Nutrition e.V., Immanuel Krankenhaus Berlin

www.vegmed.org

\section{MÄRZ 2021}

Frankfurt, 09.-13. März 2021

Schmerz- und Palliativtag 2021

ONLINE

Deutsche Gesellschaft für Schmerzme-

dizin e.V.

www.schmerz-und-palliativtag.de

Krakau, Polen,

14.-19. März 2021

VI. Congress of Chinese and

Integrative Medicine

ONLINE

Polskie Towarzystwo Chińskiej Medycyny

i Akupunktury

www.itcmkrakow.pl/kongres.html

Freudenstadt, 17.-21. März 2021

140. ZAEN-Kongress

Zentralverband der Ärzte für Naturheilverfahren und Regulationsmedizin e.V. www.zaen.org

\section{APRIL 2021}

Wien, Österreich, 10.-11. April 2021

Jahreskongress der ÖGA

8. Johannes Bischko Symposium

Hormone - STATE OF THE ART meets

TCM

Österreichische Gesellschaft für

Akupunktur (ÖGA)

www.tcmsymposium.at

Erfurt, 15.-18. April 2021

39. DGfAN-Kongress

Alt werden - gesund bleiben

Deutsche Gesellschaft für Akupunktur

und Neuraltherapie e. V. (DGfAN)

www.dgfan.de
Hamburg, 17.-18. April 2021

4. Qigong-Kongress

Philosophie- und Praxistage

Verband für Turnen und Freizeit (VTF)

www.qigong-kongress.de

Hamburg, 22.-24. April 2021

Sports, Medicine and Health Summit HYBRID

49. Deutscher Sportärztekongress

9th Congress of Exercise in Medicine

Europe

10. Internationaler Hamburger Sport-

Kongress

8. Symposium AG Diabetes Sport und

Bewegung

www.sports-medicine-health-summit.de

Hamburg, 24. April 2021

SYMPOSIUM Sport

Deutsche Ärztegesellschaft für

Akupunktur e. V. in Kooperation mit dem

Deutschen Sportärztekongress

www.daegfa.de

\section{MAI 2021}

Rothenburg o.d.T., 11.-15. Mai 2021

52. TCM Kongress

HYBRID

Arbeitsgemeinschaft für Klassische

Akupunktur und TCM e.V. (AGTCM)

www.tcm-kongress.de

Bad Nauheim, 13. Mai 2021

Jahrestagung der DÄGfA

eingebettet in die 35 . Internationale

Akupunktur-Woche in Bad Nauheim

Deutsche Ärztegesellschaft für

Akupunktur e.V. (DÄGfA)

www.daegfa.de

\section{JUNI 2021}

Pöllauberg, Österreich, 03.-05. Juni 2021

24. Jahressymposium der OGKH

Österreichische Gesellschaft für

Kontrollierte Akupunktur (OGKA)

www.ogka.at/jahressymposium

Sofia, Bulgarien, 07.-17. Juni 2021

14th International Conference on

Daoist Studies

Daoism and Literature: Myths, Stories,

Novels, Poetry

www.daoistconference.info

München, 10.-13. Juni 2021

15. Internationale Münchner

Akupunkturtage

Kooperationsveranstaltung der DÄGfA

mit der Klinik für Anaesthesiologie am

Klinikum der LMU München

www.daegfa.de

München, 12-13. Juni 2021

SYMPOSIUM Akupunktur in der

Palliativmedizin

Deutsche Ärztegesellschaft für

Akupunktur e.V. (DÄGfA)

www.daegfa.de

\section{JULI 2021}

Heidelberg, 01.-03. Juli 2021

6. Heidelberger Tagung Neural-

therapie

neuraltherapie-heidelberg.de

Athen, Griechenland, 02.-04. Juli 2021

34th ICMART World Congress on

Medical Acupuncture

International Council of Acupuncture

and Related Techniques

www.www.icmart2020.org

\section{SEPTEMBER 2021}

Köln, 04.-05. September 2021

SYMPOSIUM Akupunktur und TCM in der HNO-Heilkunde

Deutsche Ärztegesellschaft für

Akupunktur e.V. in Kooperation mit der HNO-Klinik der Uniklinik Köln und der

Klinik und Poliklinik für HNO-Heilkunde des Universitätsklinikums Dresden

www.daegfa.de

Bernried am Starnberger See

11. September 2021

Wieviele Gürtelleitbahnen gibt es?

Neue Erkenntnisse aus archäologi-

chen Funden in China

Chinesische Gesellschaft für Qigong

Yangshen e.V.

www.qigong-yangsheng.de

Freudenstadt, 22.-26. September 2021

141. ZAEN-Kongress

Zentralverband der Ärzte für Naturheilverfahren und Regulationsmedizin e.V. www.zaen.org

Graz, Österreich

23.-25. September 2021

18. Internationaler TCM Kongress

Körper und Seele in der TCM

Österreichische Gesellschaft für kontrol-

lierte Akupunktur und TCM (OGKA)

www.tcmkongress.at

\section{NOVEMBER 2021}

London, UK,

05.-07. November 2021

13th European Congress for

Integrative Medicine

"Transforming Healthcare Together"

www.ecim-congress.org

Solothurn, Schweiz,

18.-20. November 2021

14. ASA-Kongress

Association Schweizer Ärztegesellschaft für Akupunktur und Chinesische Medizin

(ASA)

www.asa-tcmkongress.ch

\section{MÄRZ 2022}

Mannheim, 18.-19. März 2022

Geburtshilfe \& TCM im Dialog

Jubiläumskongress, Forum für den

Dialog in der Chinesischen Medizin www.tcm-im-dialog.de

Freudenstadt, 23.-27. März 2022

142. ZAEN-Kongress

Zentralverband der Ärzte für Naturheilverfahren und Regulationsmedizin e.V. www.zaen.org

\section{MAI 2022}

Phoenix, Arizona, USA, 24.-27. Mai 2022 International Congress on Integrative Medicine \& Health

Academic Consortium for Integrative

Medicine \& Health

www.icimh.org

\section{SEPTEMBER 2022}

Tutzing, 09.-11. September 2022

6. Kongress der SMS

Chinesische Medizin im klinischen

Alltag - Grundlagen, Anwendung \&

Wissenschaft

Internationale Gesellschaft für

chinesische Medizin (SMS)

www.tcm.edu

Montreal, Quebec, Kanada,

10.-14. September 2022

6th International Fascia Research

Congress

www.fasciacongress.org/2021-congress/

Freudenstadt, 21.-25. September 2022 143. ZAEN-Kongress

Zentralverband der Ärzte für Naturheilverfahren und Regulationsmedizin e. V. www.zaen.org 